\title{
Some statistical aspects of the spinor field Fermi-Bose duality
}

\author{
V.M. Simulikł, I.Yu. Krivsky, I.L. Lamer \\ Institute of Electron Physics of the National Academy of Sciences of Ukraine, \\ 21 Universitetska St., 88000 Uzhgorod, Ukraine
}

Received June 25, 2012, in final form September 14, 2012

\begin{abstract}
The structure of 29-dimensional extended real Clifford-Dirac algebra, which has been introduced in our paper Phys. Lett. A, 2011, 375, 2479, is considered in brief. Using this algebra, the property of Fermi-Bose duality of the Dirac equation with nonzero mass is proved. It means that Dirac equation can describe not only the fermionic but also the bosonic states. The proof of our assertion based on the examples of bosonic symmetries, solutions and conservation laws is given. Some statistical aspects of the spinor field Fermi-Bose duality are discussed.
\end{abstract}

Key words: spinor field, symmetry, group-theoretical analysis, supersymmetry, Foldy-Wouthuysen representation, Clifford-Dirac algebra, Fermi-Bose duality

PACS: 11.30-z., 11.30.Cp., 11.30.j

\section{Introduction}

Some new statistical aspects of the Dirac equation are considered. The Fermi-Bose (FB) duality of the spinor field has been originally mentioned by L. Foldy [1]. An extended consideration has been given in [2]. P. Garbaczewski proved [2] that the Fock space $\mathscr{H}^{\mathrm{F}}\left(\mathrm{H}^{3, \mathrm{M}}\right)$ over the quantum mechanical space $\mathrm{L}_{2}\left(\mathrm{R}^{3}\right) \otimes \mathrm{C}^{\otimes \mathrm{M}}$ of a particle, which is described by the field $\phi: \mathrm{M}(1, \mathrm{~N}) \rightarrow \mathrm{C}^{\otimes N}$, allows one to fulfill the dual FB quantization of the field $\phi$ in $\mathscr{H}^{\mathrm{F}}$. Both the canonical commutation relations (CCR) and the anticommutation relations (CAR) were used to realize the above mentioned quantization. Moreover, for both types of quantization, the uniqueness of the vacuum in $\mathscr{H}^{\mathrm{F}}$ was proved. The dual FB quantization was illustrated by various examples and in the spaces $\mathrm{M}(1, \mathrm{~N})$ of arbitrary dimensions. The massless spinor field was considered in detail [2].

In our publications, the consideration of the FB duality conception of the field was extended by applying the group-theoretical approach to the problem (we often referred to the FB duality as the relationship between the fields of integer and half-integer spins, see e.g. [3-7]). As the first step, we have considered in detail the case of massless Dirac equation. Both Fermi and Bose local representations of the universal covering $\mathscr{P} \supset \mathscr{L}=\mathrm{SL}(2, \mathrm{C})$ of proper ortochronous Poincaré group $\left.\mathrm{P}_{+}^{\uparrow}=\mathrm{T}(4) \times\right) \mathrm{L}_{+}^{\uparrow} \supset \mathrm{L}_{+}^{\uparrow}=\mathrm{SO}(1,3)$, with respect to which the Dirac equation is invariant, were found. The same was realized [6] for the slightly generalized original Maxwell equations, in which the complex valued 4-object $\mathscr{E}(x)=E(x)-\mathrm{i} H(x)$ of field strengths is the tensor-scalar $(s=1,0) \mathscr{P}$-covariant. In general, we have proved the existence of bosonic symmetries, solutions and conservation laws for a massless Dirac equation [3-7]. Thus, a systematic investigation of the bosonic properties of a massless Dirac equation was carried out.

In our investigations, we followed the authors of a number of papers, in which they considered the problem of the relationship between the Dirac and Maxwell equations starting from the very origin of quantum mechanics [8-18]. However, the authors of these papers considered the simplest example of a free, massless Dirac equation and its relation to the Maxwell equations. Interest to such problems has grown after the investigations [19, 20] of a physically meaningful case (i.e., mass is nonzero and the

\footnotetext{
*E-mail: vsimulik@gmail.com
} 
interaction potential is nonzero too) and following our own research steps [21-23] in the same direction. Unfortunately, only stationary Dirac and Maxwell equations were considered.

In another approach [24-30], the quadratic relations between the fermionic and bosonic amplitudes were found and applied. In our papers [3-7, 21-23] and herein we discuss the linear relations between fermionic and bosonic amplitudes.

Our results were further developed and employed by the authors of [31-38], where the references to the above mentioned papers of ours were made. Nevertheless, the general case, where the mass in the Dirac equation is not equal to zero, is still open for investigations and considerations.

Only recently [39-42] we were able to extend our consideration to a Dirac equation with a nonzero mass. The important step was as follows. We started with the Foldy-Wouthuysen (FW) [43] representation of the Dirac equation, and the results for a standard Dirac equation were found as a consequence of the FW transformation. In our papers [39-42], bosonic representations of universal covering $\mathscr{L}=$ SL(2,C) of proper ortochronous Lorentz group $\mathrm{L}_{+}^{\dagger}=\mathrm{SO}(1,3)$ were found, with respect to which the Dirac and FW equations with nonzero mass are invariant. The main results herein are the bosonic spin $(1,0)$ representations of Poincaré group $\mathscr{P}$, with respect to which these equations are invariant. These results were proved based on the 64 dimensional extended real Clifford-Dirac (ERCD) algebra and 29 dimensional proper ERCD algebra, which were taken into consideration in [39-42] and essentially generalized the standard 16 dimensional Clifford-Dirac (CD) algebra.

Here we consider (i) the dual (fermionic and bosonic) symmetries [39-42] of the FW [43] and Dirac equations with nonzero mass, (ii) continue the construction of bosonic solutions [44] of these equations and (iii) demonstrate the existence of both Fermi and Bose conservation laws for a spinor field. Thus, we present the third level proof of the FB duality of the Dirac equation. Moreover, the statistical analysis of the Dirac model of the spin 1/2 particle doublet description is presented. We prove that such an analysis can be successful only if one uses the standard quantum-mechanical probability amplitudes distribution with respect to the eigenvalues of complete sets of experimental observable quantum-mechanical physical values. We presented in detail the corresponding quantum-mechanical stationary complete sets of operators of FB physical quantities. This allows us to demonstrate the statistical aspect of the spinor field FB duality.

For our purposes we use the mathematical formalism of the ERCD algebra and proper ERCD algebra [39-42].

In section 2, the necessary notations and definitions are presented.

In section 3, the 29-dimensional proper extended real Clifford-Dirac algebra [40-42], which is the mathematical basis of our consideration, is presented in brief.

In section 4, the bosonic spin $s=(1,0)$ symmetry [40-42] of the FW and Dirac equations with nonzero mass is briefly considered as the first step in our proof of the Dirac equation FB duality.

In section 5, we continue to construct the bosonic [44] spin $s=(1,0)$ multiplet solutions of the FW and Dirac equations with nonzero mass. This is the second step in our proof of the Dirac equation FB duality. In section 6, the FB duality of the spinor field is demonstrated based on the example of the existence of both Fermi and Bose series of conservation laws for this field (i.e., the third step of our proof).

In section 7 , the statistical aspects of the spinor field FB duality are considered.

In section 8, brief general conclusions are formulated.

\section{Notations and definitions}

The system of units $\hbar=c=1$ and metric $g=\left(g^{\mu v}\right)=(+---), a^{\mu}=g^{\mu v} a_{v}$, are taken. The Greek indices are changed in the region $0,1,2,3 \equiv \overline{0,3}$, Latin $-\overline{1,3}$, the summation over the twice repeated index is implied. The Dirac $\gamma^{\mu}$ matrices in the standard Pauli-Dirac (PD) representation are used. Our consideration is fulfilled in the rigged Hilbert space $\mathrm{S}^{3,4} \subset \mathrm{H}^{3,4} \subset \mathrm{S}^{3,4 *}$, where $\mathrm{H}^{3,4}$ is given by

$$
\mathrm{H}^{3,4}=\mathrm{L}_{2}\left(\mathrm{R}^{3}\right) \otimes \mathrm{C}^{\otimes 4}=\left\{\phi=\left(\phi^{\mu}\right): \mathrm{R}^{3} \rightarrow \mathrm{C}^{\otimes 4} ; \int \mathrm{d}^{3} x|f(t, \vec{x})|^{2}<\infty\right\}
$$

and symbol ' $*$ ' in $\mathrm{S}^{3,4 *}$ means, that the space of Schwartz generalized functions $\mathrm{S}^{3,4 *}$ is conjugated to the Schwartz test function space $S^{3,4}$ by the corresponding topology. For more details see [41]. 
We consider the ordinary CD algebra to be the algebra of $4 \times 4$ Dirac matrices in the standard PD representation in terms of the standard $2 \times 2$ Pauli matrices.

For the purposes dealing with physics it is useful to consider the corresponding groups an algebras with real parameters (e.g., the parameters $a=\left(a^{\mu}\right), \omega=\left(\omega^{\mu v}\right)$ of the translations and rotations for the group $\left.\mathrm{P}_{+}^{\uparrow}\right)$. Therefore, corresponding generators are anti-Hermitian. The mathematical correctness of such a choice of generators is verified in [45, 46].

\section{Proper extended real Clifford-Dirac algebra}

We consider the standard 16-dimensional CD algebra of the matrices to be a real one and add the imaginary unit $\mathrm{i}=\sqrt{-1}$ together with the operator $\hat{C}$ of complex conjugation (the involution operator in the space $\mathrm{H}^{3,4}$ ) into the set of the $\mathrm{CD}$ algebra possible generators. This enabled us to extend the standard CD algebra up to the 64-dimensional extended real CD algebra (i.e., ERCD algebra of [40-42]). Here, the subalgebras of the ERCD algebra are considered in brief. The most important are the representations in $\mathrm{C}^{\otimes 4} \subset \mathrm{H}^{3,4}$ of the 29-dimensional proper ERCD algebra SO(8) spanned on the orts

$$
\gamma^{1}, \quad \gamma^{2}, \quad \gamma^{3}, \quad \gamma^{4}=\gamma^{0} \gamma^{1} \gamma^{2} \gamma^{3}, \quad \gamma^{5}=\gamma^{1} \gamma^{3} \hat{C}, \quad \gamma^{6}=\mathrm{i} \gamma^{1} \gamma^{3} \hat{C}, \quad \gamma^{7}=\mathrm{i} \gamma^{0},
$$

where $\gamma^{0}=\left|\begin{array}{ll}1 & 0 \\ 0 & -1\end{array}\right|, \gamma^{k}=\left|\begin{array}{ll}0 & \sigma^{k} \\ -\sigma^{k} & 0\end{array}\right|$ and $\sigma^{k}$ are the standard Pauli matrices. The generators (2) satisfy the anticommutation relations [1]

$$
\gamma^{\mathrm{A}} \gamma^{\mathrm{B}}+\gamma^{\mathrm{B}} \gamma^{\mathrm{A}}=-2 \delta^{\mathrm{AB}}, \quad \mathrm{A}, \mathrm{B}=\overline{1,7},
$$

and the generators of the proper ERCD algebra $\alpha^{\widetilde{A} \widetilde{B}}=2 s^{\widetilde{A} \widetilde{B}}$ (together with the unit ort, $4 \times 4$ matrix $\mathrm{I}_{4}$, we have 29 independent orts $\mathrm{I}_{4}, \alpha^{\widetilde{\mathrm{A}} \widetilde{\mathrm{B}}}=2 s^{\widetilde{\mathrm{A}} \widetilde{\mathrm{B}}}$ )

$$
s^{\widetilde{\mathrm{A}} \widetilde{\mathrm{B}}}=\left\{s^{\mathrm{AB}}=\frac{1}{4}\left[\gamma^{\mathrm{A}}, \gamma^{\mathrm{B}}\right], s^{\mathrm{A} 8}=-s^{8 \mathrm{~A}}=\frac{1}{2} \gamma^{\mathrm{A}}\right\}, \quad \widetilde{\mathrm{A}}, \widetilde{\mathrm{B}}=\overline{1,8}
$$

satisfy the commutation relations of $\mathrm{SO}(8)$ algebra

$$
\left[s^{\widetilde{\mathrm{A}} \widetilde{\mathrm{B}}}, s^{\widetilde{\mathrm{C}} \widetilde{\mathrm{D}}}\right]=\delta^{\tilde{\mathrm{A}} \widetilde{\mathrm{C}}} s^{\widetilde{\mathrm{B}} \widetilde{\mathrm{D}}}+\delta^{\widetilde{\mathrm{C}} \widetilde{\mathrm{B}}} s^{\widetilde{\mathrm{D}} \widetilde{\mathrm{A}}}+\delta^{\widetilde{\mathrm{B}} \widetilde{\mathrm{D}}} s^{\widetilde{\mathrm{A}} \widetilde{\mathrm{C}}}+\delta^{\widetilde{\mathrm{D}} \widetilde{\mathrm{A}}} s^{\widetilde{\mathrm{C}} \widetilde{\mathrm{B}}}
$$

In particular, the proper ERCD algebra SO(8), given by the 29 orts [4, is our [40-42] direct generalization of the standard 16-dimensional CD algebra. It is also the basis for our dual FB consideration of a spinor field, which enabled us to prove the additional bosonic properties of this field. For physical applications, we consider the realizations of the proper ERCD algebra in the field space $S^{*}(M(1,3)) \otimes C^{\otimes 4} \equiv S^{4,4 *}$ of the Schwartz generalized functions and in the quantum mechanical Hilbert space $\mathrm{H}^{3,4}$ 1). These realizations are found with the help of transformations $V^{+} \mathrm{SO}(8) V^{-}, v \mathrm{SO}(8) v$, where the operators of transformations have the following form

$$
V^{ \pm} \equiv \frac{ \pm \mathrm{i} \vec{\gamma} \cdot \vec{\nabla}+\widehat{\omega}+m}{\sqrt{2 \widehat{\omega}(\widehat{\omega}+m)}}, \quad v=\left|\begin{array}{ll}
\mathrm{I}_{2} & 0 \\
0 & \hat{C} \mathrm{I}_{2}
\end{array}\right|, \quad \widehat{\omega} \equiv \sqrt{-\Delta+m^{2}}, \quad \vec{\nabla} \equiv\left(\partial_{\ell}\right), \quad \mathrm{I}_{2}=\left|\begin{array}{ll}
1 & 0 \\
0 & 1
\end{array}\right| .
$$

Furthermore, the realizations of the proper ERCD algebra for bosonic fields are presented.

We take into consideration the ERCD algebra (64 orts) and the proper ERCD algebra (29 orts) into the FW representation of the spinor field [43] (the advantages in comparison with the standard Dirac equation in definitions of coordinate, velocity and spin operators are well known from [43]). In this representation, the equation for the spinor field (the FW equation) has the following form

$$
\left(\partial_{0}+\mathrm{i} \gamma^{0} \widehat{\omega}\right) \phi(x)=0, \quad x \in \mathrm{M}(1,3), \quad \phi \in \mathrm{H}^{3,4}
$$

and is linked with the Dirac equation

$$
\left(\partial_{0}+\mathrm{i} H\right) \psi(x)=0, \quad H \equiv \vec{\alpha} \cdot \vec{p}+\beta m ; \quad \vec{\alpha} \equiv \gamma^{0} \vec{\gamma}, \quad \beta \equiv \gamma^{0}
$$


by the FW transformation $V^{ \pm}$

$$
\phi(x)=V^{-} \psi(x), \quad \psi(x)=V^{+} \phi(x), \quad V^{+} \gamma^{0} \widehat{\omega} V^{-}=\vec{\alpha} \cdot \vec{p}+\beta m .
$$

Herein below, the ERCD algebra and the proper ERCD algebra (4) are essentially used in our proofs of bosonic properties of the Dirac and FW equations. The proper ERCD algebra has 29 independent orts presented in (4). In comparison with 16 independent orts of standard CD algebra, we can operate now with additional elements. These additional generators of $\mathrm{SO}(8)$ algebra enabled us to prove the additional bosonic symmetries of the FW and Dirac equations [39-42] and to construct additional bosonic solutions of these equations (44] and section 5 below). Moreover, the anticommutation relations (3) were used in calculations.

\section{Bosonic spin $s=(1,0)$ symmetry of the Foldy-Wouthuysen and Dirac equations}

An example of the construction of an important bosonic symmetry of the FW and Dirac equation is under consideration. A fundamental assertion is that subalgebra SO(6) of the proper ERCD algebra (4), which is determined by the operators

$$
\begin{gathered}
\left\{\mathrm{I}, \alpha^{\overline{\mathrm{A}} \overline{\mathrm{B}}}=2 s^{\overline{\mathrm{A}} \overline{\mathrm{B}}}\right\}, \quad \overline{\mathrm{A}}, \overline{\mathrm{B}}=\overline{1,6}, \\
\left\{s^{\overline{\mathrm{A}} \overline{\mathrm{B}}}\right\}=\left\{s^{\overline{\mathrm{A}} \overline{\mathrm{B}}} \equiv \frac{1}{4}\left[\gamma^{\overline{\mathrm{A}}}, \gamma^{\overline{\mathrm{B}}}\right]\right\}
\end{gathered}
$$

is the algebra of invariance of the Dirac equation in the FW representation (7) (in (11) the six matrices $\left\{\gamma^{\bar{A}}\right\}=\left\{\gamma^{1}, \gamma^{2}, \gamma^{3}, \gamma^{4}, \gamma^{5}, \gamma^{6},\right\}$ are known from (2). Algebra SO(6) contains two different realizations of $\mathrm{SU}(2)$ algebra for the spin $\mathrm{s}=1 / 2$ doublet. By taking the sum of the two independent sets of SU(2) generators from (11), one can obtain the SU(2) generators of spin $s=(1,0)$ multiplet, which generate the transformation of the invariance of the FW equation (7). These operators can be presented in the following form

$$
\vec{s} \equiv\left(\breve{s}^{j}\right)=\left(\breve{s}_{m n}\right)=\frac{1}{2}\left(\breve{\gamma}^{2} \breve{\gamma}^{3}-\breve{\gamma}^{0} \breve{\gamma}^{2} \breve{C}, \breve{\gamma}^{3} \breve{\gamma}^{1}+\breve{i} \breve{\gamma}^{0} \breve{\gamma}^{2} \breve{C}, \breve{\gamma}^{1} \breve{\gamma}^{2}-\breve{i}\right)
$$

where the corresponding orts of the ERCD algebra in bosonic representation are given by

$$
\begin{aligned}
& \breve{\gamma}^{0}=\left|\begin{array}{ll}
\sigma^{3} & 0 \\
0 & \sigma^{1}
\end{array}\right|, \quad \breve{\gamma}^{1}=\frac{1}{\sqrt{2}}\left|\begin{array}{cccc}
0 & 0 & 1 & -1 \\
0 & 0 & \mathrm{i} & \mathrm{i} \\
-1 & \mathrm{i} & 0 & 0 \\
1 & \mathrm{i} & 0 & 0
\end{array}\right|, \quad \breve{\gamma}^{2}=\frac{1}{\sqrt{2}}\left|\begin{array}{cccc}
0 & 0 & -\mathrm{i} & \mathrm{i} \\
0 & 0 & -1 & -1 \\
-\mathrm{i} & 1 & 0 & 0 \\
\mathrm{i} & 1 & 0 & 0
\end{array}\right| \\
& \breve{\gamma}^{3}=-\left|\begin{array}{ll}
\sigma^{2} & 0 \\
0 & \mathrm{i} \sigma^{2}
\end{array}\right| \hat{C}, \quad \breve{\gamma}^{4}=\left|\begin{array}{cc}
\mathrm{i} \sigma^{2} & 0 \\
0 & -\sigma^{2}
\end{array}\right| \hat{C}, \quad \breve{\gamma}^{5}=\frac{1}{\sqrt{2}}\left|\begin{array}{cccc}
0 & 0 & -1 & -1 \\
0 & 0 & \mathrm{i} & -\mathrm{i} \\
1 & \mathrm{i} & 0 & 0 \\
1 & -\mathrm{i} & 0 & 0
\end{array}\right|, \\
& \breve{\gamma}^{6}=\frac{1}{\sqrt{2}}\left|\begin{array}{cccc}
0 & 0 & -\mathrm{i} & -\mathrm{i} \\
0 & 0 & 1 & -1 \\
-\mathrm{i} & -1 & 0 & 0 \\
-\mathrm{i} & 1 & 0 & 0
\end{array}\right|, \quad \breve{\gamma}^{7}=\gamma^{7}=\mathrm{i} \gamma^{0}, \quad \breve{i}=\left|\begin{array}{ll}
\mathrm{i} \sigma^{3} & 0 \\
0 & -\mathrm{i} \sigma^{1}
\end{array}\right|, \quad \breve{C}=\left|\begin{array}{ll}
\sigma^{3} & 0 \\
0 & \mathrm{I}_{2}
\end{array}\right| \hat{C} .
\end{aligned}
$$

The spin operators (12) of SU(2) algebra, which commute with the operator $\partial_{0}+\mathrm{i} \gamma^{0} \widehat{\omega}$ of the FW equation (7), can also be presented in an explicit form

$$
\breve{s}^{1}=\frac{1}{\sqrt{2}}\left|\begin{array}{cccc}
0 & 0 & \mathrm{i} \hat{C} & 0 \\
0 & 0 & -\hat{C} & 0 \\
-\mathrm{i} \hat{C} & \hat{C} & 0 & 0 \\
0 & 0 & 0 & 0
\end{array}\right|, \quad \breve{s}^{2}=\frac{1}{\sqrt{2}}\left|\begin{array}{cccc}
0 & 0 & \hat{C} & 0 \\
0 & 0 & -\mathrm{i} \hat{C} & 0 \\
-\hat{C} & \mathrm{i} \hat{C} & 0 & 0 \\
0 & 0 & 0 & 0
\end{array}\right|, \quad \breve{s}^{3}=\left|\begin{array}{cccc}
-\mathrm{i} & 0 & 0 & 0 \\
0 & \mathrm{i} & 0 & 0 \\
0 & 0 & 0 & 0 \\
0 & 0 & 0 & 0
\end{array}\right| .
$$


The calculation of the Casimir operator for the SU(2) generators (14) gives the following result

$$
\vec{s}^{2}=-1(1+1) \times\left|\begin{array}{cc}
\mathrm{I}_{3} & 0 \\
0 & 0
\end{array}\right|
$$

Transition from the fundamental representation A of the ERCD algebra to the bosonic representation $\mathrm{B}$ is fulfilled $\mathrm{B}=W \mathrm{~A} W^{-1}$ using the operator $W$ :

$$
W=\frac{1}{\sqrt{2}}\left|\begin{array}{cccc}
\sqrt{2} & 0 & 0 & 0 \\
0 & 0 & \mathrm{i} \sqrt{2} \hat{C} & 0 \\
0 & -\hat{C} & 0 & 1 \\
0 & -\hat{C} & 0 & -1
\end{array}\right|, \quad W^{-1}=\left|\begin{array}{cccc}
\sqrt{2} & 0 & 0 & 0 \\
0 & 0 & -\hat{C} & -\hat{C} \\
0 & \mathrm{i} \sqrt{2} \hat{C} & 0 & 0 \\
0 & 0 & 1 & -1
\end{array}\right|, \quad W W^{-1}=W^{-1} W=\mathrm{I}_{4} \text {. }
$$

Based on the spin operators (12), (14), the bosonic spin $(1,0)$ representation of the Poincaré group $\mathscr{P}$ is constructed. It is easy to show (after our consideration in [43] and above) that generators

$$
p_{0}=-\mathrm{i} \gamma_{0} \widehat{\omega}, \quad p_{n}=\partial_{n}, \quad j_{l n}=x_{l} \partial_{n}-x_{n} \partial_{l}+\breve{s}_{l n}, \quad j_{0 k}=x_{0} \partial_{k}+\mathrm{i} \gamma_{0}\left\{x_{k} \widehat{\omega}+\frac{\partial_{k}}{2 \widehat{\omega}}+\frac{(\overrightarrow{\tilde{s}} \times \vec{\partial})_{k}}{\widehat{\omega}+m}\right\}
$$

of group $\mathscr{P}$ commute with the operator of the FW equation (7) and satisfy the commutation relations of the Lie algebra of the group $\mathscr{P}$ in a manifestly covariant form. In the space $\mathrm{H}^{3,4}$, the operators (16) generate a unitary $\mathscr{P}$ representation differing from the fermionic $\mathscr{P}$ F-generators according to equations (D-64)-(D-67) in [1], i.e., the bosonic $\mathscr{P}^{\mathrm{B}}$ representation of the group $\mathscr{P}$, with respect to which the FW equation (7) is invariant. For the generators (16), the Casimir operators have the following form:

$$
p^{\mu} p_{\mu}=m^{2}, \quad W^{\mathrm{B}}=w^{\mu} w_{\mu}=m^{2} \overrightarrow{\vec{s}}^{2}=-1(1+1) m^{2}\left|\begin{array}{cc}
\mathrm{I}_{3} & 0 \\
0 & 0
\end{array}\right| .
$$

Hence, according to the Bargman-Wigner classification, here we consider the spin $s=(1,0)$ representation of the group $\mathscr{P}$.

The corresponding bosonic spin $s=(1,0)$ symmetries of the Dirac equation 8 can be found from the generators (16) using the FW operator (6) in bosonic representation, i.e., $W V^{ \pm} W^{-1}$.

More complete and detailed consideration of the bosonic symmetries of the FW and Dirac equation was given in $40-42]$.

\section{Bosonic spin $s=(1,0)$ multiplet solution of the Foldy-Wouthuysen and Dirac equations}

Here, as the next step in FB duality investigation, we consider the bosonic solution of the Dirac (FW) equation. A bosonic solution of the FW equation (7) is found completely similarly to the procedure of construction of standard fermionic solution. Thus, the bosonic solution is determined by some stationary diagonal complete set of operators of bosonic physical quantities for the spin $s=(1,0)$-multiplet in the FW representation, e.g., by the set “momentum-spin projection $\breve{s}^{3}$ ”:

$$
\left(\vec{p}=-\vec{\nabla}, \quad \vec{s}^{3}\right),
$$

where the spin operators $\vec{s}$ and $\breve{s}^{3}$ for the spin $s=(1,0)$-multiplet are given in 12, 14). The fundamental solutions of equation (7), which are the common eigensolutions of the bosonic complete set (18), have the following form

$$
\varphi_{\vec{k} \mathrm{r}}^{-}(t, \vec{x})=\frac{1}{(2 \pi)^{3 / 2}} \mathrm{e}^{-\mathrm{i} k x} \mathrm{~d}_{\mathrm{r}}, \quad \varphi_{\vec{k} \hat{\mathrm{r}}}^{+}(t, \vec{x})=\frac{1}{(2 \pi)^{3 / 2}} \mathrm{e}^{\mathrm{i} k x} \mathrm{~d}_{\hat{\mathrm{r}}}, \quad k x=\omega t-\vec{k} \cdot \vec{x},
$$

where $\mathrm{d}_{\alpha}=\left(\delta_{\alpha}^{\beta}\right)$ are the Cartesian orts in the space $\mathrm{C}^{\otimes 4} \subset \mathrm{H}^{3,4}$, numbers $\mathrm{r}=(1,2), \hat{\mathrm{r}}=(3,4)$ mark the eigenvalues $(+1,-1,0, \underline{0})$ of the operator $\breve{s}^{3}$ from (12), (14). 
The bosonic solutions of equation (7) are the generalized states belonging to the space $\mathrm{S}^{3,4 *}$; they form a complete orthonormalized system of bosonic states. Therefore, any bosonic physical state of the FW field $\phi$ from the dense in $\mathrm{H}^{3,4}$ manifold $\mathrm{S}^{3,4}$ (the general bosonic solution of the equation (7)) is uniquely presented in the following form

$$
\phi_{(1,0)}(x)=\frac{1}{(2 \pi)^{3 / 2}} \int \mathrm{d}^{3} k\left[\xi^{\mathrm{r}}(\vec{k}) \mathrm{d}_{\mathrm{r}} \mathrm{e}^{-\mathrm{i} k x}+\xi^{* \mathrm{r}}(\vec{k}) \mathrm{d}_{\hat{\mathrm{r}}} \mathrm{e}^{\mathrm{i} k x}\right]
$$

where $\xi(\vec{k})$ are the coefficients of the expansion of bosonic solution of the FW equation (7) with respect to the Cartesian basis (19). The relationships of amplitudes $\xi(\vec{k})$ with quantum-mechanical bosonic amplitudes $b(\vec{k})$ of probability distribution according to the eigenvalues of the stationary diagonal complete set of operators of quantum-mechanical bosonic $s=(1,0)$-multiplet are presented by

$$
\xi^{1}=b^{1}, \quad \xi^{2}=-\frac{1}{\sqrt{2}}\left(b^{3}+b^{4}\right), \quad \xi^{3}=-\mathrm{i} b^{2}, \quad \xi^{4}=\frac{1}{\sqrt{2}}\left(b^{3}-b^{4}\right) ; \quad b^{1,2,3,4}(\vec{k}) \equiv b^{+,-, 0, \underline{0}}(\vec{k})
$$

where the 4 amplitudes $b^{1,2,3,4}(\vec{k})$ are the quantum-mechanical momentum-spin amplitudes with the eigenvalues $(+1,-1,0, \underline{0})$ of the projection $\breve{s}^{3}$ on the axe 3 of the quantum mechanical spin $s=(1,0)$ multiplet operator $\overrightarrow{\vec{s}}$, respectively (the last eigenvalue $\underline{0}$ is related to the proper zero spin). Thus, if $\phi_{(1,0)}(x) \in$ $\mathrm{S}^{3,4}$, then the bosonic amplitudes $\xi(\vec{k})$ belong to the Schwartz complex-valued test function space too.

Moreover, the set $\left\{\phi_{(1,0)}(x)\right\}$ of solutions 20 is invariant, in particular, with respect to the unitary bosonic representation of the group $\mathscr{P}$, which is determined by the generators (16) and Casimir operators 17. Therefore, the Bargman-Wigner analysis of the Poincaré symmetry of the set $\left\{\phi_{(1,0)}(x)\right\}$ of solutions (20) manifestly demonstrates that this is the set of Bose-states $\phi_{(1,0)}$ of the field $\phi$, i.e., the $s=(1,0)$ multiplet states. Hence, the existence of bosonic solutions of the FW equation is proved.

In terms of quantum-mechanical momentum-spin amplitudes $b^{\alpha}(\vec{k})$ from $(21)$, the bosonic spin $(1,0)-$ multiplet solution $\psi=V^{+} \phi$ of the Dirac equation (8) is presented by

$$
\begin{aligned}
\psi_{(1,0)}(x)= & \frac{1}{(2 \pi)^{3 / 2}} \int \mathrm{d}^{3} k\left\{\mathrm{e}^{-\mathrm{i} k x}\left[b^{1} v_{1}^{-}(\vec{k})-\frac{1}{\sqrt{2}}\left(b^{3}+b^{4}\right) v_{2}^{-}(\vec{k})\right]\right. \\
& \left.+\mathrm{e}^{\mathrm{i} k x}\left[i b^{* 2} v_{1}^{+}(\vec{k})+\frac{1}{\sqrt{2}}\left(b^{* 3}-b^{* 4}\right) v_{2}^{+}(\vec{k})\right]\right\}
\end{aligned}
$$

where the 4-component spinors are the same as in the Dirac theory of the fermionic doublet

$$
v_{\mathrm{r}}^{-}(\vec{k})=N\left|\begin{array}{c}
(\widehat{\omega}+m) \mathrm{d}_{\mathrm{r}} \\
(\vec{\sigma} \cdot \vec{k}) \mathrm{d}_{\mathrm{r}}
\end{array}\right|, \quad v_{\mathrm{r}}^{+}(\vec{k})=N\left|\begin{array}{c}
(\vec{\sigma} \cdot \vec{k}) \mathrm{d}_{\mathrm{r}} \\
(\widehat{\omega}+m) \mathrm{d}_{\mathrm{r}}
\end{array}\right|, \quad N \equiv \frac{1}{\sqrt{2 \widehat{\omega}(\widehat{\omega}+m)}}, \quad \mathrm{d}_{1}=\left|\begin{array}{c}
1 \\
0
\end{array}\right|, \quad \mathrm{d}_{2}=\left|\begin{array}{l}
0 \\
1
\end{array}\right| .
$$

The well known (i.e., standard) Fermi solution of the Dirac equation for the spin $s=1 / 2$ doublet has the following form

$$
\psi(x)=\frac{1}{(2 \pi)^{3 / 2}} \int \mathrm{d}^{3} k\left[\mathrm{e}^{-\mathrm{i} k x} a_{\mathrm{r}}^{-}(\vec{k}) v_{\mathrm{r}}^{-}(\vec{k})+\mathrm{e}^{\mathrm{i} k x} a_{\mathrm{r}}^{+}(\vec{k}) v_{\mathrm{r}}^{+}(\vec{k})\right],
$$

where the physical sense of the amplitudes $a_{\mathrm{r}}^{-}(\vec{k}), a_{\mathrm{r}}^{+}(\vec{k})$ is explained in 47].

All the above assertions concerning the FB duality of the spinor field are valid both in FW and PD representation, i.e., for both FW (7) and Dirac (8) equations. The transition between FW and PD representations is fulfilled by the FW transformation (6).

\section{The Fermi-Bose conservation laws for the spinor field}

Let us briefly note the FB conservation laws (CL) for the spinor field. It is preferable to calculate them in the FW (nonlocal PD) representation too. In FW representation, the Fermi spin $\vec{s}=\left(s^{23}, s^{31}, s^{12}\right)$ from (11) (together with the "boost spin") is the independent symmetry operator for the FW equation. The orbital angular momentum and pure Lorentz angular momentum (the carriers of external statistical degrees of freedom) are independent symmetry operators in this representation too (one can also find 
the corresponding independent spin and angular momentum symmetries in the PD representation for the Dirac equation, but the corresponding operators are essentially nonlocal). Hence, one obtains 10 Poincaré and 12 additional (3 spin, 3 pure Lorentz spin, 3 angular momentum, 3 pure angular momentum) CL.

Therefore, in the FW representation, one can easily find 22 fermionic and 22 bosonic CL. The division into bosonic and fermionic set is caused by the existence of FB symmetries and solutions. Indeed, if substitution of bosonic $\mathscr{P}$ generators $q(16)$ and bosonic solutions $[20$ into the Noether formula $Q=\int \mathrm{d}^{3} x \phi^{\dagger}(x) q \phi(x)$ is made, then automatically the bosonic CL for $s=(1,0)$-multiplet are obtained. The standard substitution of the corresponding well known fermionic generators and solutions presents fermionic CL.

We briefly illustrate the difference in fermionic and bosonic CL based on the example of the corresponding spin conservation. For a fermionic spin

$$
\vec{s}=\left(s_{23}, s_{31}, s_{12}\right) \equiv\left(s^{\ell}\right)=\frac{1}{2}\left|\begin{array}{cc}
\vec{\sigma} & 0 \\
0 & \vec{\sigma}
\end{array}\right| \rightarrow s_{z} \equiv s^{3}=\frac{1}{2}\left|\begin{array}{cccc}
1 & 0 & 0 & 0 \\
0 & -1 & 0 & 0 \\
0 & 0 & 1 & 0 \\
0 & 0 & 0 & -1
\end{array}\right|
$$

and for a bosonic spin 12), 14, the CL are given by

$$
\begin{aligned}
& S_{m n}^{\mathrm{F}}=\int \mathrm{d}^{3} x \phi^{\dagger}(x) s_{m n} \phi(x)=\int \mathrm{d}^{3} k A^{\dagger}(\vec{k}) s_{m n} A(\vec{k}), \\
& S_{m n}^{\mathrm{B}}=\int \mathrm{d}^{3} x \phi^{\dagger}(x) \breve{s}_{m n} \phi(x)=\int \mathrm{d}^{3} k B^{\dagger}(\vec{k}) \breve{s}_{m n} B(\vec{k}),
\end{aligned}
$$

where

$$
A(\vec{k})=\operatorname{column}\left(a_{+}^{-}, a_{-}^{-}, a_{-}^{*+}, a_{+}^{*+}\right), \quad B(\vec{k})=\operatorname{column}\left(b^{1}, b^{2}, b^{* 3}, b^{* 4}\right) .
$$

We present these CL in terms of quantum-mechanical Fermi and Bose amplitudes. All integral conserved quantities have an explicit quantum-statistical form.

\section{The Fermi-Bose duality and the Fermi-Bose statistics}

An adequate statistical quantum-mechanical sense of the coefficients $a_{\mathrm{r}}^{-}(\vec{k}), a_{\mathrm{r}}^{+}(\vec{k})$ in the expansion (24) over the basis solutions (23) of the Dirac equation is found in the same way but with the help of transition $\phi(x)=V^{-} \psi(x)$ [9], (6) to the FW representation [43]. Indeed, the statistical sense of the FW field $\phi(x)$ is evidently related to the statistical sense of the particle-antiparticle doublet in relativistic canonical quantum mechanics [43, 48] of this doublet. It is shown in [43] that

$$
\phi=\left|\begin{array}{c}
\phi^{-} \\
0
\end{array}\right|+\left|\begin{array}{c}
0 \\
\phi^{*+}
\end{array}\right|
$$

where $\phi^{\mp}(x)$ are the relativistic quantum-mechanical wave functions of the particle-antiparticle doublet.

The solution of the FW equation (7) expanded over the eigenvectors of quantum-mechanical fermionic stationary diagonal complete set of operators (momentum $\vec{p}$, projection $s^{3}$ of the spin $\vec{s}^{\text {quant.-mech. }}$ and sign of the charge $g=-\gamma^{0}$ ) has the following form

$$
\phi(x)=\frac{1}{(2 \pi)^{3 / 2}} \int \mathrm{d}^{3} k\left\{\mathrm{e}^{-\mathrm{i} k x}\left[a_{+}^{-}(\vec{k}) \mathrm{d}_{1}+a_{-}^{-}(\vec{k}) \mathrm{d}_{2}\right]+\mathrm{e}^{\mathrm{i} k x}\left[a_{-}^{*+}(\vec{k}) \mathrm{d}_{3}+a_{+}^{*+}(\vec{k}) \mathrm{d}_{4}\right]\right\},
$$

where the coefficients of expansion $a_{+}^{-}(\vec{k}), a_{-}^{-}(\vec{k}), a_{-}^{+}(\vec{k}), a_{+}^{+}(\vec{k})$ denote the statistical quantum-mechanical amplitudes of probability distribution over the eigenvalues of the above mentioned fermionic stationary complete set of operators. The 4-columns $\mathrm{d}_{\alpha}=\left(\delta_{\alpha}^{\beta}\right)$ are the Cartesian orts in the space $\mathrm{C}^{\otimes 4} \subset \mathrm{H}^{3,4}$. In order to obtain the most adequate and obvious statistical quantum-mechanical interpretation of the amplitudes 
and solutions, the spin projection operator in a complete set (momentum $\vec{p}$, projection $s^{3}$ of the spin $\vec{s}^{\text {quant.-mech. }}$ and sign of the charge $g=-\gamma^{0}$ ) is taken in the quantum-mechanical form [48]

$$
\vec{s}^{\text {quant. }- \text { mech. }}=\frac{1}{2}\left|\begin{array}{ll}
\vec{\sigma} & 0 \\
0-C \vec{\sigma} C
\end{array}\right| \rightarrow s_{z}^{\text {quant.-mech. }} \equiv s^{3}=\frac{1}{2}\left|\begin{array}{cccc}
1 & 0 & 0 & 0 \\
0 & -1 & 0 & 0 \\
0 & 0 & -1 & 0 \\
0 & 0 & 0 & 1
\end{array}\right|
$$

rather than in the canonical field theory form 25. The statistical sense of the amplitudes is conserved in the solution $\left[\psi(x)=V^{+} \phi(x)\right.$ (9), (6)]

$$
\begin{aligned}
\psi(x)= & \frac{1}{(2 \pi)^{3 / 2}} \int \mathrm{d}^{3} k\left\{\mathrm{e}^{-\mathrm{i} k x}\left[a_{+}^{-}(\vec{k}) \nu_{1}^{-}(\vec{k})+a_{-}^{-}(\vec{k}) \nu_{2}^{-}(\vec{k})\right]\right. \\
& \left.+\mathrm{e}^{\mathrm{i} k x}\left[a_{-}^{*+}(\vec{k}) \nu_{1}^{+}(\vec{k})+a_{+}^{*+}(\vec{k}) \nu_{2}^{+}(\vec{k})\right]\right\}
\end{aligned}
$$

of the Dirac equation $(8)$ in its standard local representation. The amplitudes $a_{+}^{-}(\vec{k}), a_{-}^{-}(\vec{k}), a_{-}^{+}(\vec{k}), a_{+}^{+}(\vec{k})$ in the fermionic solutions (30) and (32) of the FW and Dirac equations are one and the same. Thus, $a_{+}^{-}(\vec{k})$, $a_{-}^{-}(\vec{k})$ are the quantum-mechanical momentum-spin amplitudes of the particle with charge $-e$ and eigenvalues of spin projection $+1 / 2$ and $-1 / 2 ; a_{-}^{+}(\vec{k}), a_{+}^{+}(\vec{k})$ are the quantum-mechanical momentum-spin amplitudes of the antiparticle with charge $+e$ and eigenvalues of spin projection $-1 / 2$ and $+1 / 2$, respectively.

Statistical quantum mechanical sense of the bosonic amplitudes $b^{\alpha}(\vec{k})$ of bosonic solution (22) of the Dirac equation $(8)$ is found in the same way and is explained in section 5 in the process of constructing this solution.

The relationship between the fermionic $a_{+}^{-}(\vec{k}), a_{-}^{-}(\vec{k}), a_{-}^{+}(\vec{k}), a_{+}^{+}(\vec{k})$ and bosonic $b^{1,2,3,4}(\vec{k}) \equiv b^{+,-, 0,0}(\vec{k})$ amplitudes in one and the same (arbitrarily fixed) physical state of FB dual field $\psi$ is presented by the unitary operator $U$ in the following form:

$$
\left|\begin{array}{c}
a_{+}^{-} \\
a_{-}^{-} \\
a_{-}^{+} \\
a_{+}^{+}
\end{array}\right|=\frac{1}{\sqrt{2}}\left|\begin{array}{cccc}
\sqrt{2} & 0 & 0 & 0 \\
0 & 0 & -1 & -1 \\
0 & -\mathrm{i} \sqrt{2} & 0 & 0 \\
0 & 0 & 1 & -1
\end{array}\right|\left|\begin{array}{c}
b^{+} \\
b^{-} \\
b^{0} \\
b^{0}
\end{array}\right|, \quad\left|\begin{array}{c}
b^{+} \\
b^{-} \\
b^{0} \\
b^{0}
\end{array}\right|=\frac{1}{\sqrt{2}}\left|\begin{array}{cccc}
\sqrt{2} & 0 & 0 & 0 \\
0 & 0 & \mathrm{i} \sqrt{2} & 0 \\
0 & -1 & 0 & 1 \\
0 & -1 & 0 & -1
\end{array}\right|\left|\begin{array}{c}
a_{+}^{-} \\
a_{-}^{-} \\
a_{-}^{+} \\
a_{+}^{+}
\end{array}\right|
$$

Relationships (33) follow directly from the comparison of the solutions (22) and (32).

Note that the set of fermionic solutions $\left\{\psi^{\mathrm{F}}\right\}$ (32) of the Dirac equation is invariant with respect to the well known induced fermionic $\mathscr{P}^{\mathrm{F}}$ representation of the Poincaré group $\mathscr{P}$ [49], see also formula (19) in the paper [41]). The set of bosonic solutions $\left\{\psi^{\mathrm{B}}\right\}$ (22) of the Dirac equation is invariant with respect to the induced bosonic $\mathscr{P}^{\mathrm{B}}$ representation of the Poincaré group $\mathscr{P}$ (formula (21) in the paper [42]). However, the relationships (33) between the fermionic $a_{+}^{-}(\vec{k}), a_{-}^{-}(\vec{k}), a_{-}^{+}(\vec{k}), a_{+}^{+}(\vec{k})$ and bosonic $b^{1,2,3,4}(\vec{k}) \equiv b^{+,-, 0, \underline{0}}(\vec{k})$ amplitudes do not change in any inertial frame of references.

\section{Conclusions}

The 64 dimensional ERCD and 29 dimensional proper ERCD algebras, which have been put into consideration in 40-42], are useful generalizations of the standard 16 dimensional CD algebra. Application thereof enabled us to prove the existence of additional bosonic symmetries, solutions and conservation laws for the spinor field, the Foldy-Wouthuysen and the Dirac equations. The investigation of the spinor field in the Foldy-Wouthuysen representation has the sense and purpose of its own. This representation is of great interest in itself, especially in connection with the recent result [50] by V. Neznamov, who developed the formalism of quantum electrodynamics in the Foldy-Wouthuysen representation, see also the results in [51]. The property of the Fermi-Bose duality of the Dirac equation (both in the FoldyWouthuysen and the Pauli-Dirac representations), which proof was started in [39-42], where the bosonic symmetries of this equation were found, is demonstrated herein on the next level, i.e., on the level of the existence of the spin $(1,0)$ bosonic solutions of the equation under consideration and corresponding 
bosonic conservation laws. Similarly, the fermionic spin $s=1 / 2$ properties for the Maxwell equations both with nonzero and zero mass can be proved (see e.g., the procedure given in [6]).

In any case, we do not change the main well known postulates and theory of the Fermi-Bose statistics. Our results have another, principally new meaning. In our approach, the Fermi-Bose duality of the spinor field found in [2] is proved based on the examples of the existence of bosonic symmetries (section 4) and solutions (section 5) of the Dirac equation with nonzero mass together with the confirmation of the bosonic conservation laws (section 6) for the spinor field. This opens up new possibilities for applying the Dirac equation to the description of bosonic states. Thus, the property of the Fermi-Bose duality of the Dirac equation that was proved in our publications [39-42] and in the present paper does not break the Fermi statistics for fermions (with the Pauli principle) and Bose statistics for bosons (with Bose condensation). We have never mixed up the Fermi and Bose statistics as well. Our assertion is as follows. One can apply with equal success both Fermi and Bose statistics for one and the same Dirac equation and for one and the same spinor field, i.e., the Dirac equation can describe both fermionic and bosonic states.

\section{References}

1. Foldy L., Phys. Rev., 1956, 102, 568; doi 10.1103/PhysRev.102.568

2. Garbaczewski P., Int. J. Theor. Phys., 1986, 25, 1193; doi/10.1007/BF00668690

3. Simulik V., Theor. Math. Phys., 1991, 87, 386; doi 10.1007/BF01016578

4. Krivsky I., Simulik V., Theor. Math. Phys., 1992, 90, 265; doi 10.1007/BF01036532

5. Simulik V., Krivsky I., Adv. Appl. Cliff. Alg., 1998, 8, 69; doi 10.1007/BF03041926

6. Simulik V., Krivsky I., Rep. Math. Phys., 2002, 50, 315; doi 10.1016/S0034-4877(02)80062-3

7. Simulik V., Krivsky I., Electromag. Phenomena, 2003, 50, 103.

8. Darwin C., Proc. R. Soc. London, Ser. A, 1928, 118, 654; doi 10.1098/rspa.1928.0076

9. Mignani R., Recami E., Badlo M., Lett. Nuovo Cimento, 1974, 11, 568; doi 10.1007/BF02812391

10. Laporte O., Uhlenbeck G.E., Phys. Rev., 1931, 37, 1380; doi 10.1103/PhysRev.37.1380

11. Oppenheimer J.R., Phys. Rev., 1931, 38, 725; doi 10.1103/PhysRev.38.725

12. Good R.H., Phys. Rev., 1957, 105, 1914; doi 10.1103/PhysRev.105.1914

13. Borhgardt A.A., Zh. Eksp. Teor. Fiz., 1958, 34, 334.

14. Lomont J.S., Phys. Rev., 1958, 111, 1710; doi 10.1103/PhysRev.111.1710.

15. Moses H.E., Phys. Rev., 1959, 113, 1670; doi 10.1103/PhysRev.113.1670

16. Da Silveira A., Z. Naturforsch. A, 1979, 34, 646.

17. Gianetto E., Lett. Nuovo Cimento, 1985, 44, 140; doi 10.1007/BF02746912

18. Ljolje K., Fortschr. Phys., 1988, 36, 9; doi 10.1002/prop.2190360103

19. Sallhofer H., Z. Naturforsch. A, 1978, 33, 1379.

20. Sallhofer H., Z. Naturforsch. A, 1990, 45, 1361.

21. Simulik V.M., Ukr. Math. J. 1997, 49, 1075; doi 10.1007/BF02528753

22. Simulik V.M., Krivsky I.Yu., Adv. Appl. Cliff. Alg., 1997, 7, 25; doi 10.1007/BF03041213

23. Simulik V.M., The electron as a system of classical electromagnetic and scalar fields. In: What is the electron? Ed. by Simulik V.M., Apeiron, Montreal, 2005, pp. 109-134.

24. Campolattaro A., Int. J. Theor. Phys., 1980, 19, 99; doi 10.1007/BF00669764

25. Campolattaro A., Int. J. Theor. Phys., 1990, 29, 141; doi 10.1007/BF00671324

26. Daviau C., Ann. Fond. L. de Brogl., 1989, 14, 273.

27. Daviau C., Lochak J., Ann. Fond. L. de Brogl., 1991, 16, 43.

28. Rodrigues W. Jr., De Oliveira E.C., Int. J. Theor. Phys., 1990, 29, 397; doi 10.1007/BF00674439

29. Rodrigues W. Jr.,Vaz J. Jr., Found. Phys., 1998, 28, 789; doi 10.1023/A:1018854004954

30. Keller J., Adv. Appl. Cliff. Alg., 1999, 9, 309; doi 10.1007/BF03042383

31. Keller J., In: Proc. of the Int. Conf. "The theory of electron”, Mexico, 1995; Adv. Appl. Cliff. Alg., 1997, 7, 25; doi 10.1007/BF03041213

32. Kruglov S., Ann. Fond. L. de Brogl., 2001, 26, 725.

33. Xuegang Y., Shuna Z., Qiunan H., Adv. Appl. Cliff. Alg., 2001, 11, 27; doi 10.1007/BF03042037

34. Grudsky S.M., Khmelnitskaya K.V., Kravchenko V.V. J. Phys. A, 2004, 37, 4641; doi 10.1088/0305-4470/37/16/013

35. Armour R.C. Jr., Found. Phys., 2004, 34, 815; doi 10.1023/B:FOOP.0000022188.90097.10

36. Varlamov V., Int. J. Mod. Phys. A, 2005, 20, 4095; doi 10.1142/S0217751X05025048

37. Rozzi T., Mencarelli D., Pierantoni L., IEEE: Trans. Microwave Theor. Techn., 2009, 57, 2907; doi 10.1109/TMTT.2009.2034225 
38. Okninski A., Symmetry, 2012, 4, 427; doi 10.3390/sym4030427.

39. Simulik V.M., Krivsky I.Yu., Preprint arXiv:math-ph/0908.3106, 2009.

40. Simulik V.M., Krivsky I.Yu., Rep. Nat. Acad. Sci. of Ukraine, 2010, 5, 82.

41. Krivsky I.Yu., Simulik V.M., Condens. Matter Phys., 2010, 4, 43101; doi 10.5488/CMP.13.43101

42. Simulik V.M., Krivsky I.Yu., Phys. Lett. A, 2011, 375, 2479; doi 10.1016/j.physleta.2011.03.058

43. Foldy L., Wouthuysen S., Phys. Rev., 1950, 78, 29; doi 10.1103/PhysRev.78.29

44. Simulik V.M., Krivsky I.Yu., Zajac T.M., Preprint arXiv:math-ph/1112.5712, 2011.

45. Elliott J.P., Dawber P.J., Symmetry in Physics, Vol.1., Macmillian Press, London, 1979.

46. Wybourne B.G., Classical Groups for Physicists, John Wiley and Sons, New York, 1974.

47. Bogoliubov N.N., Shirkov D.V., Introduction to the Theory of Quantized Fields, John Wiley and Sons, New York, 1980.

48. Krivsky I.Yu., Simulik V.M., Zajac T.M., Lamer I.L., In: Proc. of the 14-th Int. Conf. "Mathematical methods in electromagnetic theory”, Kharkiv, Ukraine, 2012, 28-30.

49. Thaller B, The Dirac Equation, Berlin, Springer, 1992.

50. Neznamov V.P., Phys. Part. Nucl., 2006, 37, 86; doi 10.1134/S1063779606010023.

51. Neznamov V.P., Silenko A.J., J. Math. Phys., 2009, 50, 122302; doi 10.1063/1.3268592

\title{
Деякі статистичні аспекти Фермі-Бозе дуалізму спінорного поля
}

\author{
В.М. Симулик, І.Ю. Кривський, І.Л. Ламер
}

Інститут електронної фізики, Національна академія наук України, вул. Університетська, 21, 88000 Ужгород, Україна

Коротко розглядається структура 29-вимірної розширеної дійсної алгебри Кліффорда-Дірака, яка була введена в розгляд у нашій публікації Phys. Lett. A, 2011, 375, 2479. На основі цієї алгебри доводиться властивість Фермі-Бозе дуалізму рівняння Дірака з ненульовою масою. Це означає, що рівняння Дірака може описувати не лише ферміонні, але й бозонні стани. Доведення дається на прикладах наявності бозонних симетрій, розв'язків та законів збереження. Розглянуто деякі статистичні аспекти Фермі-Бозе дуалізму спінорного поля

Ключові слова: спінорне поле, симетрія, теоретико-груповий аналіз, суперсиметрія, представлення Фолді-Ваутхайзена, алгебра Кліффорда-Дірака, Фермі-Бозе дуалізм 